\title{
Science funding in low- and middle-income countries: the example of vaccine research
}

\author{
RH Branston ${ }^{1}$ and JAG Whitworth ${ }^{1}$
}

\section{There has been a recent increase in funding sources and overall commitment to tackle global health issues and reduce morbidity and mortality. The Wellcome Trust, the UK's largest charity, has a long track record of funding research in global health and has developed a new research strategy to tackle these issues. Here, we describe the Wellcome Trust's approach to funding researchers in low- and middle-income countries with a focus on how our funding portfolio supports vaccine delivery and development, with specific reference to mucosal immunity.}

\section{INTRODUCTION}

There is currently an increased interest in global health among politicians and policy makers, funding agencies both governmental and philanthropic, and in civil societies around the world. The focus of "Research for Health," as articulated at the recent Global Ministerial Forum in Bamako, ${ }^{1}$ highlights the relevance of research to improve well-being of human populations in low- and middle-income countries. The recent G-Finder report has documented the extent of funding on neglected disease research and development ${ }^{2}$ and indicates that though such research is conducted by a wide variety of organizations including those in academia, public institutions, and private companies, it is funded by a relatively small number of organizations with increasing proportions being provided by private and philanthropic organizations rather than governments, even those of wealthy countries. The report notes the tendency to focus on developing new drugs and vaccines for a small number of diseases rather than on the wider agenda of diagnostics, platform technologies, and developing country-specific products needed for many high-burden, highmortality diseases.

Using the Wellcome Trust as an example of a major medical research charity, we discuss how we have responded to these recent changes in circumstance and attitude toward global health. Although we have had a long-term interest in funding of tropical medicine research, starting with an award to Henry Foy in 1938 to study malaria control in Greece, today we fund thousands of researchers in dozens of countries around the world and have expanded the proportion of our international funding to around $15 \%$ of total expenditure. Our main strategic goals are

- to broaden the research base for global health research by increasing the number of individual researchers, and institutions and systems within which they can flourish;

- to support research that will benefit health - and the opportunity to make a difference is nowhere clearer than in resource-poor settings;

- to support networks and partnerships focused on the problems of low- and middle-income countries where there are shared aims and a synergy of expertise.

It is people who conduct scientific research and we aim to identify and fund those scientists who are asking the highest quality research questions wherever they may be in the world.

Several of the Millennium Development Goals focus on health, and in many cases the most effective way to prevent morbidity and mortality is to vaccinate susceptible populations. Vaccinology and research into vaccine development and deployment are, therefore, important components of our science-funding portfolio, and we use multiple mechanisms in flexible ways to support research in these fields.

\section{A FUNDERS APPROACHTO VACCINOLOGY}

The path to successful vaccine delivery can be considered in a step-wise manner (see Figure 1). It starts with basic research to understand effective immune mechanisms and to develop candidate vaccine constructs, moves through pre-clinical and clinical trials and onto health systems and health policy

${ }^{1}$ Science Funding Division, The Wellcome Trust, 215, Euston Road, London, UK. Correspondence: RH Branston (r.branston@wellcome.ac.uk) 


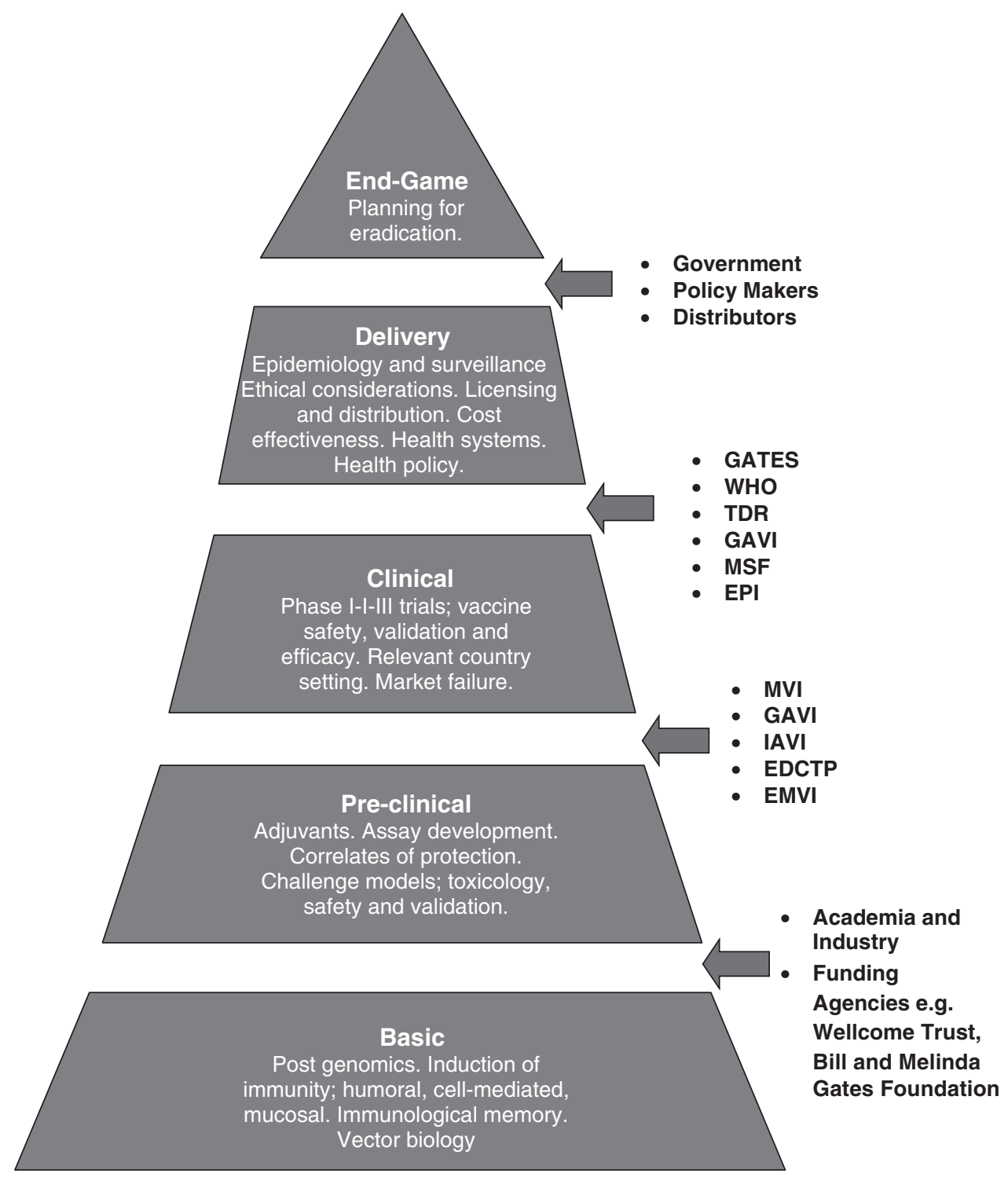

Figure $1 \mathrm{~A}$ schematic diagram indicating the steps in the vaccine development process for infectious disease.

research into delivery in populations and finally end-game issues relating to elimination and eradication. Successful vaccine development and delivery will require commitment to networks and partnerships from many players including academia, industry, public-private partnerships, government, policy makers, as well as donor and funding agencies. We have examined the steps involved in vaccine delivery and particularly focus on how to overcome the gaps and hurdles that occur along each step of the developmental pathway.

The basic scientific challenges to vaccine development include improving molecular understanding of target antigens and developing related diagnostic tools, improving understanding of the various arms of the immune system in protection, and understanding how to elicit broad and persistent immune responses. Historically most successes in immunization have been achieved through the induction of protective antibodies, whereas a major challenge facing vaccinology today is the induction of broad-based persistent T-cell immunity and memory B cells, through CD4 + and $\mathrm{CD} 8+$ cell responses. Mucosal surfaces are major portals of entry for important pathogens, such as infections of the lower respiratory tract including tuberculosis, diarrheal diseases, HIV, and other sexually transmitted infections. Mucosal infections are particular problems in low- and middle-income countries where sanitation and health education are lacking, and as such represent a major target for vaccine development. However, a better understanding of the mucosal immune system is required to facilitate further mucosal vaccine development. Our understanding is limited, and necessitates research in developing countries to ensure appreciation of real-world issues such as co-existing malnutrition and concurrent infections, especially chronic infections causing inflammation and altered immune responses, such as helminths and HIV infections. Oral immunization using living organisms to stimulate mucosal responses have been used for some time 
for enteric diseases such as typhoid and polio, and now attempts to induce mucosal responses with inactivated strains are being pursued, such as for oral inactivated cholera vaccine. Primeboost strategies have been shown to be immunogenic for certain pathogens like $\mathrm{H} 5 \mathrm{~N} 1$ virus, but there is also a need to develop single-dose vaccines that elicit stronger and longer-lasting mucosal immune responses to avoid the logistic difficulties of boosters and repeat dose schedules. Advances in genomics and post-genomic approaches should greatly increase the potential for studies of the immune system and more specifically will facilitate the further identification of target antigens for vaccines and diagnostics. Investigation of delivery routes, for example sublingual delivery, and a better understanding of the compartmentalization of mucosal responses are required, in addition to studies to develop new antigen delivery systems and better ways to measure mucosal immune responses. Most of these issues can be addressed through applications to our Immunology and Infectious Disease Funding Committee and our response mode mechanism of supporting Project, Programme, and Fellowship grants.

Challenges for testing candidates in preclinical studies include development of potent adjuvants, systematic methods for identification of correlates of protection, and identification of surrogate markers of efficacy and toxicity. The lack of surrogate means of testing vaccine efficacy and limited knowledge of correlates of protection limits the early assessment of many vaccines, including those for HIV, malaria, and TB. This contributes to inefficient and costly vaccine development. There are currently very few adjuvants that are licensed for use in humans, especially for mucosal vaccines, and access to these is limited because of commercial sensitivities and a lack of formulation data. As we move forward, there may also be a need for tailor-made adjuvants as the trend develops toward subunit vaccines that have often limited immunogenicity. Fortunately, there has been much interest in this area over the past few years particularly to increase access through partnerships between academia and industry. We recently issued a targeted call for research proposals in this field.

Clinical vaccine trials in developing countries are limited by the lack of infrastructure for field trial sites, the number of trained investigators in low- and middle-income countries, and market failure and political inertia worldwide. We have highlighted the need to strengthen capacity for health research in low- and middle-income countries, particularly those in Africa, ${ }^{3}$ and the Wellcome Trust has recently developed initiatives to increase research capacity at individual, institutional, and systems levels in Africa through the Health Research Capacity Strengthening Initiative, ${ }^{4-6}$ the African Institutions Initiative, ${ }^{7}$ and also through a new partnership with the Indian Government aiming to fund around 70 new fellowships for Indian scientists each year. ${ }^{8}$ Vaccines may perform differently in developing country populations where many diseases are endemic and disease exposure and earlier immunity varies; therefore, it is imperative that clinical trials are conducted in those populations that stand to gain the most from their implementation. A recent example being for the newly developed rotavirus vaccines. The Wellcome Trust does fund a small number of clinical trials, especially, in developing countries and where there is a limited commercial drive for development, through both our Technology Transfer Divisions and the Science Funding Populations and Public Health Funding Committee.

Once vaccines have been evaluated, there are major challenges for effective delivery to target populations. Epidemiological surveillance data are required to guide policy makers in making rational decisions about vaccine deployment. This requires new diagnostic and surveillance tools and strengthening of infrastructure and healthcare systems. There is some appreciation of the global burden and importance of the major tropical diseases, HIV, TB, and malaria ${ }^{9,10}$ but for many diseases, even those with high mortality and morbidity, for example cholera and typhoid, there is gross under-reporting and under-funding. Even when vaccines are deployed, supply can be insufficient, vaccine manufacturers may be too few and regulatory pressures render production increasingly difficult. The Wellcome Trust works with the World Health Organization and other policy-making and funding bodies to plan for public health threats such as influenza pandemics by documenting existing research activities and mapping out important research priorities for the future, and to support cost-benefit analyses to inform governments of the practicalities of introducing a vaccine.

The final stage represents end-game concerns and preparing for elimination and eradication. Establishing if an infectious disease has been eliminated is no easy task, especially when infection can be asymptomatic, as is the case for $90 \%$ of poliovirus infections. Certified eradication relies on excellent surveillance and laboratory diagnosis. Polio end-game issues include when to stop vaccinating, when to move from live-attenuated oral polio vaccine (which can cause vaccinederived polio) to more costly inactivated injectable polio vaccine, and the continuing need for surveillance. As diseases decline and profiles are reduced, the need for control strategies becomes less evident to policy makers and the need for public engagement and education increases.

\section{CONCLUSION}

The Wellcome Trust over the 10 years between 1995 and 2005, committed over $£ 73$ million to the area of vaccinology, supporting a wide range of science from basic research through clinical trials and public health research through multiple mechanisms of support. Of this commitment, approximately $30 \%$ of grants involved either direct or indirect funding to low- or middle-income countries. We remain committed to research for health that will improve the use of vaccines available today and identify the new vaccines that will be required in the future.

\section{DISCLOSURE}

The authors declare no conflict of interest.

C 2009 Society for Mucosal Immunology

\section{REFERENCES}

1. Bamako Ministerial Global Forum (2008). http://www.bamako2008.org/.

2. Moran, M. et al. Neglected disease research and development: how much are we really spending? PLoS Med. 6, e30 (2009). 
3. Whitworth, J.A. Strengthening capacity for health research in Africa. Lancet $\mathbf{3 7 2}$, 1590-1593 (2008).

4. Health Research Capacity Strengthening. http://www.wellcome.ac.uk/Funding/ Biomedical-science/International-funding/ Global-health-research/WTDV026103.htm

5. Nordling, L. African grant comes with no strings attached. Nat. News. http://www. nature.com/news/2009/090226/full/ news.2009.127.html.

6. Nightingale, K. Donors test pioneering funding model SciDevNet. http://www.scidev.net/en/ news/donors-test-pioneering-funding-model. html.

7. African Institutions Initiative. http://www. wellcome.ac.uk/Funding/Biomedical-science/ Grants/Other-initiatives/WTD028338.htm.
8. Wellcome Trust DBT_-India Alliance. http://www.wellcomedbt.org/index. $\mathrm{htm}$.

9. Hay, S.I. et al. A world malaria map: Plasmodium falciparum endemicity in 2007. PLoS Med. 6, e1000048 (2009).

10. The Malaria Atlas Project. http://www.map. ox.ac.uk. 